\title{
Diagnosis prediction of tumours of unknown origin using ImmunoGenius, a machine learning-based expert system for immunohistochemistry profile interpretation
}

Yosep Chong ${ }^{1,2^{*}}$ (D) Nishant Thakur ${ }^{1}$, Ji Young Lee ${ }^{1}$, Gyoyeon Hwang ${ }^{1}$, Myungjin Choi ${ }^{3}$, Yejin Kim ${ }^{4,5}$, Hwanjo $\mathrm{Yu}^{6}$ and Mee Yon $\mathrm{Cho}^{7}$

\begin{abstract}
Background: Immunohistochemistry (IHC) remains the gold standard for the diagnosis of pathological diseases. This technique has been supporting pathologists in making precise decisions regarding differential diagnosis and subtyping, and in creating personalized treatment plans. However, the interpretation of $\mathrm{IHC}$ results presents challenges in complicated cases. Furthermore, rapidly increasing amounts of $\mathrm{HC}$ data are making it even harder for pathologists to reach to definitive conclusions.

Methods: We developed ImmunoGenius, a machine-learning-based expert system for the pathologist, to support the diagnosis of tumors of unknown origin. Based on Bayesian theorem, the most probable diagnoses can be drawn by calculating the probabilities of the $\mathrm{IHC}$ results in each disease. We prepared $\mathrm{IHC}$ profile data of 584 antibodies in 2009 neoplasms based on the relevant textbooks. We developed the reactive native mobile application for iOS and Android platform that can provide 10 most possible differential diagnoses based on the IHC input.

Results: We trained the software using 562 real case data, validated it with 382 case data, tested it with 164 case data and compared the precision hit rate. Precision hit rate was 78.5, 78.0 and $89.0 \%$ in training, validation and test dataset respectively. Which showed no significant difference. The main reason for discordant precision was lack of disease-specific IHC markers and overlapping IHC profiles observed in similar diseases.

(Continued on next page)
\end{abstract}

\footnotetext{
* Correspondence: ychong@catholic.ac.kr

'Department of Hospital Pathology, College of Medicine, The Catholic

University of Korea, 271, Cheonbo-ro, Uijeongbu 11765, Gyeonggi-do,

Republic of Korea

${ }^{2}$ Postech-Catholic Biomedical Engineering institute, College of Medicine, The Catholic University of Korea, Seoul, Republic of Korea

Full list of author information is available at the end of the article
}

C C The Author(s). 2021 Open Access This article is licensed under a Creative Commons Attribution 4.0 International License, which permits use, sharing, adaptation, distribution and reproduction in any medium or format, as long as you give appropriate credit to the original author(s) and the source, provide a link to the Creative Commons licence, and indicate if changes were made. The images or other third party material in this article are included in the article's Creative Commons licence, unless indicated otherwise in a credit line to the material. If material is not included in the article's Creative Commons licence and your intended use is not permitted by statutory regulation or exceeds the permitted use, you will need to obtain permission directly from the copyright holder. To view a copy of this licence, visit http://creativecommons.org/licenses/by/4.0/ The Creative Commons Public Domain Dedication waiver (http://creativecommons.org/publicdomain/zero/1.0/) applies to the data made available in this article, unless otherwise stated in a credit line to the data. 
(Continued from previous page)

Conclusion: The results of this study showed a potential that the machine-learning algorithm based expert system can support the pathologic diagnosis by providing second opinion on $\mathrm{HC}$ interpretation based on $\mathrm{HC}$ database. Incorporation with contextual data including the clinical and histological findings might be required to elaborate the system in the future.

Keywords: Database, Expert system, Machine learning, Immunohistochemistry, Probabilistic decision tree

\section{Background}

Immunohistochemical staining (IHC) is an essential staining method for differentiating tumor origin in pathologic diagnosis. It enables to infer the origin of cells by investigating the expression of specific antigens in the tissue [1-6]. In 1941, Dr. Albert Coons developed an indirect form of immunofluorescence staining technique [1, 7]. Initially, it was designed for staining fresh tissue samples and samples were visualized by fluorescence microscopy. However, with the introduction of enzyme-conjugated antibodies and paraffinembedding, IHC became a regularly used assay in the diagnosis of pathological conditions [2-6]. Simultaneously, the role of IHC has been extended from classifying the cellular origin of tumours to the subtyping tumours, determining treatment efficacy, predicting patient prognosis (prognostic marker), and finally differentiating precancerous lesions by evaluating the molecular changes $[1-3,8]$.

However, the rapidly expanding knowledge about IHC positivity in each neoplasm often leads to conflicting interpretations in routine practices, especially in some complicated cases [9]. For example, a combination of TTF-1 (lung and thyroid), galectin-3 (100\% in papillary thyroid cancers), and napsin A (lung adenocarcinomas) is used to determine the tumour origin of a lung mass in patients with thyroid nodules [10, 11]. However, in different lung cancer subtypes, TTF-1 positivity changes from 21 to $91 \%$, and galectin-3 shows $49 \%$ positivity in the subset of lung adenocarcinomas, and napsin A shows a positivity of less than $5 \%$ in thyroid cancers, which means that the IHC results by themselves cannot exclude the rare exceptions [11-13]. The interpretation of IHC results can be biased depending on the experience and knowledge of the individual pathologists $[2,4,6]$. Presently, thousands of new antibodies and IHC staining data from various tumours are available to researchers. Over a hundred thousand studies using IHC-based assays have been published since 2000. Therefore, it is not feasible for the pathologists to memorize the expression of all the molecular markers recognized by the constantly evolving repertoire of antibodies in tumours from different tissues of origin [14].

Algorithmic approaches and standardized IHC panels for certain diagnoses have been used to solve this problem $[9,14,15]$. However, in clinical practice, each case is unique and sensitive, and generalized application of particular IHC panels in some cases can be timeconsuming and labour-intensive.

Thus, we developed an expert system using computer software, in the form of an iOS and Android mobile application-based on a machine-learning algorithm and IHC database IHC that assists pathologists in making a precise diagnosis.

\section{Methods}

This study was approved by the Institutional Review Board of the Catholic University of Korea, College of Medicine (SC17RCDI0074).

\section{Development of machine-learning algorithm using probabilistic decision tree}

Bayesian theorem is one of the main topic in the field of probability theory and statistics. This indicates a relationship for random variables between conditional probabilities and marginal probabilities. According to Bayesian theorem, the post-event probability can be calculated when the pre-event probability is given. Bayes' theorem is stated mathematically as $\mathrm{P}(\mathrm{B}) \neq 0$, where $\mathrm{A}$ and $\mathrm{B}$ are events $[16] . \mathrm{P}(\mathrm{A} \mid \mathrm{B})$ and $\mathrm{P}(\mathrm{B} \mid \mathrm{A})$ are the conditional probabilities, such that the likelihood of event $A$ occurring, given that $\mathrm{B}$ has occurred and vice versa, respectively. $\mathrm{P}(\mathrm{A})$ and $\mathrm{P}(\mathrm{B})$ are the probabilities of observing $\mathrm{A}$ and $\mathrm{B}$ independently of each other [16].

$$
P(A \mid B)=\frac{P(B \mid A) P(A)}{P(B)}
$$

IHC results are binary and the probability of positive and negative IHC in each neoplasm is empirically known by pathologists and relatively well documented in the textbooks and literature (Fig. 1). Although incidence of each neoplasm should be pre-event probability, incidence of each neoplasm varies with various other factors such as ethnicity, and we are dealing with the hypothetical probability that is only based on IHC results. In addition, the effect of pre-event probability can be too high when additional conditions are few and its effect can be low when additional conditions are many enough. 


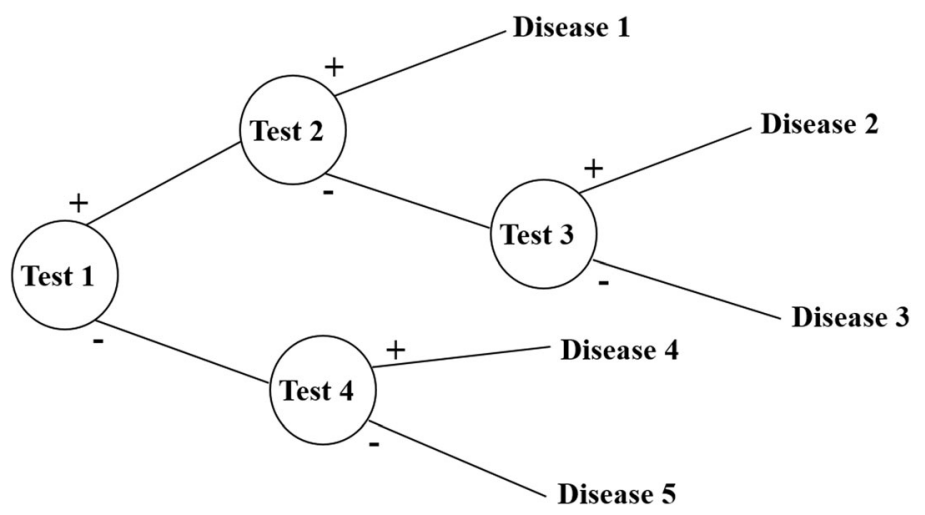

Fig. 1 Probabilistic decision tree for the machine-learning algorithm in diagnostic tests and disease

Therefore, we hypothetically supposed that the preevent probability is neglectable for computation.

Collectively, we need a database of a $2 \times 2$ table with tests, diseases, and the probability of positivity of each test for each disease. Test results obtained are binary. The probability of positivity signifies the number of positive cases among all the cases of the disease. Once the test results are obtained, the probability for each disease can be calculated by multiplying the prior probability and the probabilities of each test being positive or negative, to indicate the illness with the highest probability, by comparing post probability.

Let us take an example as in Fig. 2. Suppose that the pre-event disease probability is $30 \%$ for Disease 1, 50\% for Disease 2, and 20\% for Disease 3, and the known probability of positive results of Test $\mathrm{A}, \mathrm{B}$, and $\mathrm{C}$ for each disease is as shown in the table of Fig. 2. If we get the results of Test A, B, C as positive, negative, and positive, we can calculate post probability as the equations next to the table. As a result, the probability of Disease 3 is the highest upon the test results.

\section{Construction of IHC database}

As shown in Supplementary table 1, important textbooks on IHC such as Classification of Tumours Series (IARC, Lyon, France) and literature from World Health Organization (WHO), were used to build an IHC database based on the IHC expression profile of all tumours [4, 5, 17-28]. Over 5000 different neoplasms were recorded based on the WHO classification. Neoplasms without IHC expression profile were excluded. Differences in the IHC profile of tumour subtypes, were recorded separately from the primary type.

Each tumours IHC positivity was recorded as showed in the textbook. If there was no exact numerical value attributed to the positivity, arbitrary expressions such as "always positive", "often positive", or "rarely/ occasionally positive" were assigned. The positivity of each tumour was described as: "always": 95\%; "often": 75\%; "in about a half of cases": 50\%; "seldom": 30\%; "rarely/ occasionally": $10 \%$; and "never": $0 \%$. If the positivity differs between textbooks, the average value was used in the database. IHC database showed in Supplementary Fig. 1.

Around 600 antibody names and their synonyms used in IHC were recorded using the textbooks and reviewed with the online references Supplementary table 2.

\section{Development of ImmunoGenius, a mobile application for iOS and android}

The "ImmunoGenius" mobile application for iOS and Android was developed using NoSQL (Fig. 3) and can be accessed on iPhones, Android phones, and iPads. It is designed to search for diseases and upon selection of the illness it generates a table with the IHC

\begin{tabular}{|c|c|c|c|c|c|c|}
\hline & & Test A & Test B & Test $\mathrm{C}$ & Prior probability & Post probability \\
\hline & $\begin{array}{c}\text { Disease } 1 \\
(30 \%)\end{array}$ & $60 \%$ & $30 \%$ & $0 \%$ & $0.3 \mathrm{x}$ & $0.6 \times 0.7 \times 0.0$ \\
\hline & $\begin{array}{l}\text { Disease } 2 \\
(50 \%)\end{array}$ & $20 \%$ & $80 \%$ & $10 \%$ & $0.5 \mathrm{x}$ & $0.2 \times 0.2 \times 0.1$ \\
\hline & $\begin{array}{c}\text { Disease } 3 \\
(20 \%)\end{array}$ & $50 \%$ & $50 \%$ & $70 \%$ & $0.2 \mathrm{x}$ & $0.5 \times 0.5 \times 0.7$ \\
\hline & Test results & + & - & + & \multicolumn{2}{|c|}{$\begin{array}{c}\text { The probability of Disease } 3 \text { is the highest with the test } \\
\text { results }\end{array}$} \\
\hline
\end{tabular}


antibody names in the first row and disease name in the left column. The IHC profiles are showed in the corresponding cells designated as " ++ " for 75-100\% positivity, "+" for $50-74 \%$, "+/-" for $30-49 \%, "-/+$ " for $10-29 \%$, and "-" for $0-9 \%$ shown with graded shades (Fig. 4). Individuals can compare the different IHC profiles and add or remove the diseases and IHC antibodies to customize the table. Importantly, individuals can add their IHC results through a button on the right-hand side. Once the IHC results are inserted, the diagnosis presumption algorithm calculates the top 10 most probable diagnoses, which are shown along with the estimated probability (red numbers). The detailed user instructions and software download is available at homepage: https:// immunogenius.wixsite.com/website)

google play store: https://play.google.com/store/apps/ details?id=com.dasomx.ig\&hl=ko

you tube video: https://youtu.be/0EUQKCmAXc8

\section{Validation of diagnosis presumption algorithm using patient data}

To prove the precision of the diagnosis presumption algorithm, IHC profile data was generated for specific cases and diagnosed by pathologists using conventional methods. These were then compared with the top 10 results from the presumptive diagnoses algorithm. The IHC profile data of 1000 tumours of unknown origin (TUOs) collected between 2010 to 2017 from the Yeouido and Seoul St. Mary's Hospital, College of Medicine, The Catholic University of Korea were used in this study. Any data related to patient identification, except the original diagnosis and the IHC results, were blinded before data processing. In addition, we collected the IHC profile data of 164 TUOs for test dataset diagnosed in 2020 from the archives at Uijeongbu St. Mary's Hospital, College of Medicine, The Catholic University of Korea. TUOs were defined as the cases in clinical or pathological situation, where the immunohistochemical differential diagnosis is needed to differentiate between primary or metastatic lesions, or between variable subtypes of cancers, for confirmative diagnosis. In such cases, the histological findings alone cannot exclude the possibility of misdiagnosis or misclassification (e.g. determination of tumour origin in ascites, pleural fluid, or lymph nodes; determination of primary or metastatic lesions and pathologic subtyping in the needle biopsy samples of lung, liver, or kidney, where metastasis is common and clinicoradiologic findings are not confirmative). For training and validation, the retrieved database was divided into 6:4. The cases with inadequate IHC profiles such as the absence of markers for tumour origins, IHC less than three antibodies, inconclusive results were excluded. However, only prognostic markers such as EGFR or p53 were eliminated. Supplementary Fig. 2 showed an example of retrieved IHC profile dataset from patients. The precision of diagnosis presumption algorithm was confirmed by the inclusion of the diagnosis obtained by conventional methods in the top 10 presumptive diagnoses generated by the algorithm. It is considered to be inclusive, without significant difference in the IHC profile, between the initial and presumptive diagnosis, but the only difference in location (e.g., gastrointestinal stromal tumour of the stomach vs. small intestine). The hit rate of training and validation data was compared to prove the functionality of the algorithm. The algorithm is considered validated, if there is no statistically significant difference between the training and validation dataset. After training

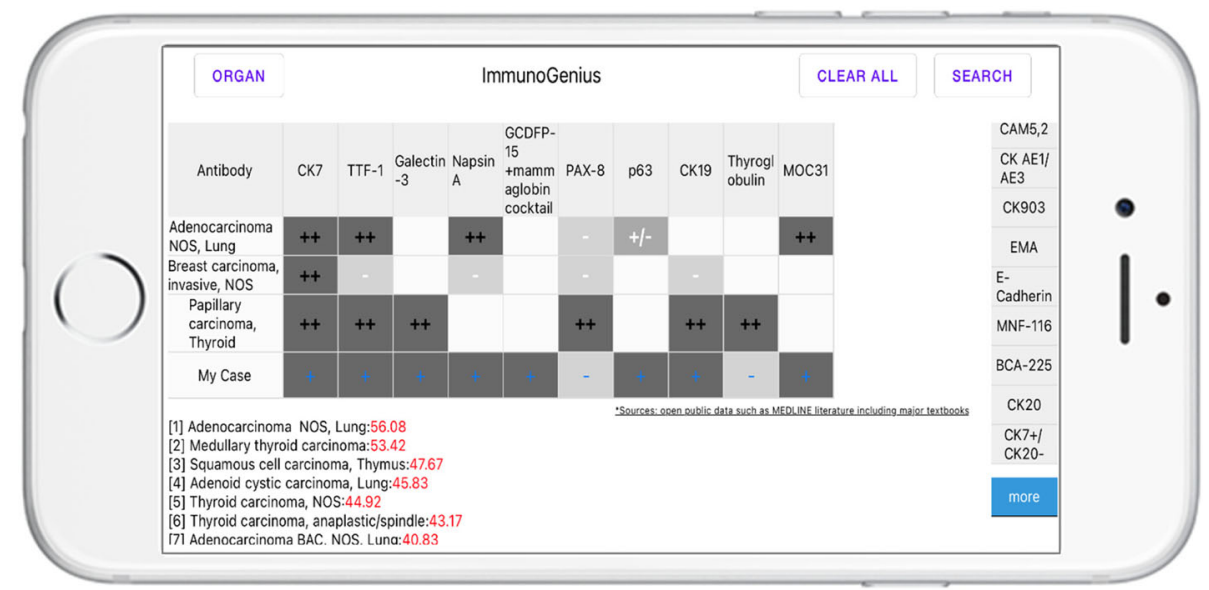

Fig. 3 The screenshot of the mobile application "ImmunoGenius" 


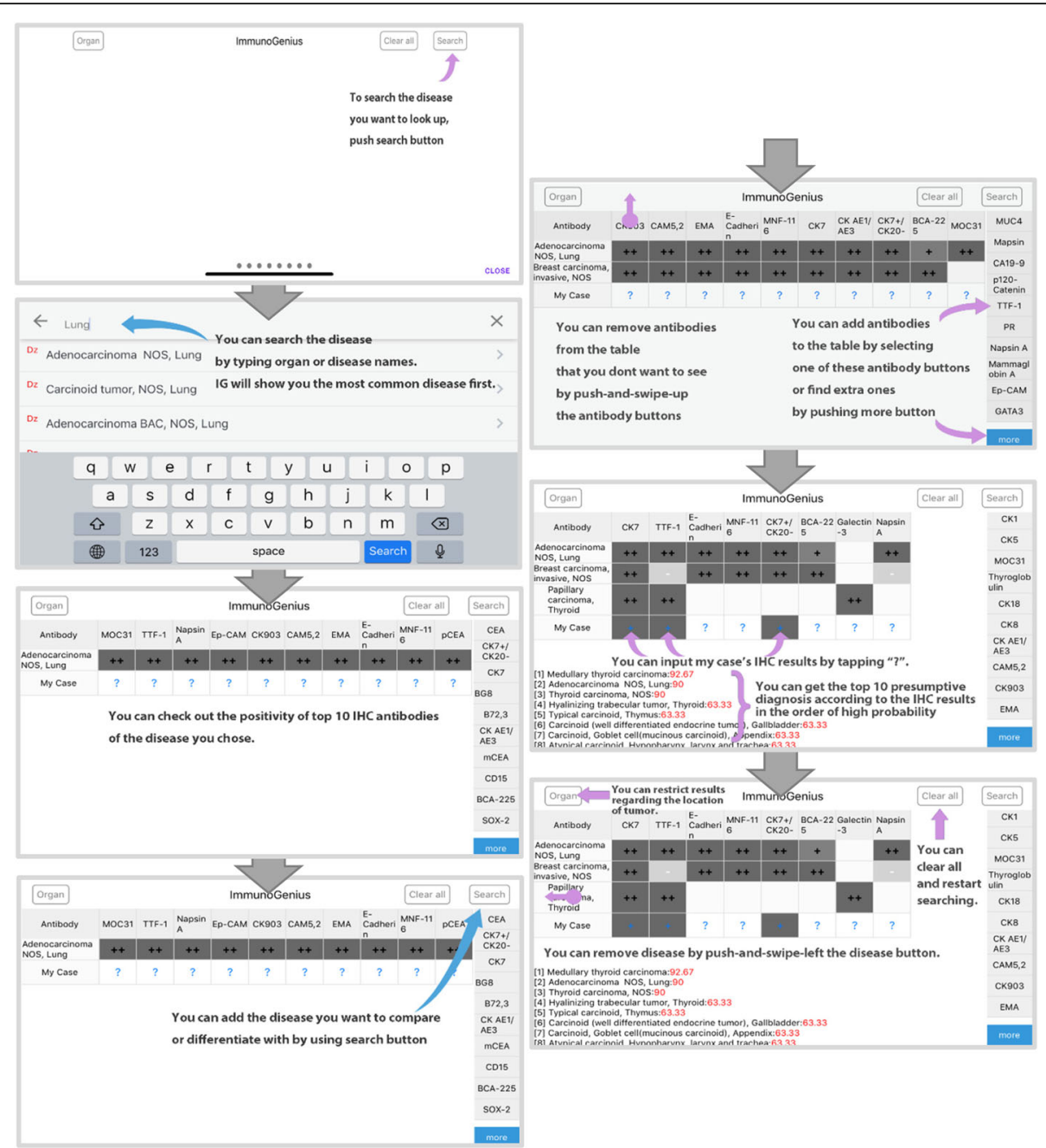

Fig. 4 Exemplary flowchart for ImmunoGenius

and validation, algorithm was tested with dataset of another institute (external validation).

\section{Statistical analysis}

Time and computer complexity were accessed by testing the mobile application. Chi-square test was used to compare the hit rate between original and presumptive diagnoses. A web-based statistical analysis ("http://webr.org") was used for statistical analysis.

\section{Results}

Construction of IHC database. Recruitment of training, validation, and test dataset

The detailed information related to 2009 different types of cancer, 584 IHC antibodies, and their IHC profiles were recorded in the IHC database. Five hundred sixtytwo cases were used for the training dataset, 382 cases were used for the validation dataset and 164 cases for test dataset.

\section{Training data}

The recruited training and validation data of the tumours were from 562 and 382 cases, respectively. On an average, 6.8 IHC antibodies (ranged 1-13) were used for diagnosis. A wide variety of tumours from 32 organs were included. The organ and the original diagnoses of the training data cases are shown in Tables 1 and 2. The common organs were lung (20.6\%), liver (9.8\%), kidney (6.6\%), stomach (6.6\%), and large intestine/rectum (5.3\%) (Table 1). Ascites and peritoneum consist of $5.7 \%$, while pleural fluid and pleura comprised of $5.2 \%$ (Table 1) of the cases. Primary carcinoma consists of $41.3 \%$ of the cases, followed by metastatic carcinoma (26.9\%), benign mesenchymal tumour (21.4\%), mild (normal) lesion (5.9\%), and malignant mesenchymal tumour (4.6\%) (Table 2). The hit rate of the presumptive diagnosis of the training data (top 10) was $78.5 \%$ (Table 3). The error rates being the highest at $30.8 \%$ in malignant mesenchymal tumours, followed by metastatic carcinoma (25.8\%), benign mesenchymal 
Table 1 The organs of the training and validation dataset of TUO

\begin{tabular}{|c|c|c|c|c|c|c|}
\hline \multirow[t]{2}{*}{ Organ } & \multicolumn{2}{|c|}{ Training data } & \multicolumn{2}{|c|}{ Validation data } & \multicolumn{2}{|c|}{ Test data } \\
\hline & No. & $\%$ & No. & $\%$ & No & $\%$ \\
\hline Ascites (cell block) & 19 & $3.4 \%$ & 11 & $2.9 \%$ & 1 & $0.6 \%$ \\
\hline Pleural fluid (cell block) & 15 & $2.7 \%$ & 12 & $3.1 \%$ & 0 & $0.0 \%$ \\
\hline Lymph node & 12 & $2.1 \%$ & 9 & $2.4 \%$ & 8 & $4.9 \%$ \\
\hline Peritoneum & 13 & $2.3 \%$ & 8 & $2.1 \%$ & 2 & $1.2 \%$ \\
\hline Pleura & 14 & $2.5 \%$ & 7 & $1.8 \%$ & 2 & $1.2 \%$ \\
\hline Lung & 116 & $20.6 \%$ & 75 & $19.6 \%$ & 26 & $15.9 \%$ \\
\hline Liver & 55 & $9.8 \%$ & 43 & $11.3 \%$ & 33 & $20.1 \%$ \\
\hline Kidney & 37 & $6.6 \%$ & 31 & $8.1 \%$ & 15 & $9.1 \%$ \\
\hline Breast & 17 & $3.0 \%$ & 11 & $2.9 \%$ & 1 & $0.6 \%$ \\
\hline Soft tissue & 38 & $6.8 \%$ & 14 & $3.7 \%$ & 6 & $3.6 \%$ \\
\hline Female genital tract including uterus and vulva, vagina & 17 & $3.0 \%$ & 12 & $3.1 \%$ & 18 & 10.1 \\
\hline Adnexa & 8 & $1.4 \%$ & 6 & $1.6 \%$ & 0 & $0.0 \%$ \\
\hline Bladder and urinary tract & 7 & $1.2 \%$ & 5 & $1.3 \%$ & 1 & $0.6 \%$ \\
\hline Adrenal gland & 10 & $1.8 \%$ & 5 & $1.3 \%$ & 1 & $0.6 \%$ \\
\hline Prostate & 15 & $2.7 \%$ & 11 & $2.9 \%$ & 0 & $0.0 \%$ \\
\hline Testis & 8 & $1.4 \%$ & 5 & $1.3 \%$ & 0 & $0.0 \%$ \\
\hline Pancreas & 7 & $1.2 \%$ & 4 & $1.0 \%$ & 3 & $1.8 \%$ \\
\hline Stomach & 37 & $6.6 \%$ & 20 & $5.2 \%$ & 9 & $5.5 \%$ \\
\hline Small intestine & 4 & $0.7 \%$ & 1 & $0.3 \%$ & 5 & $3.0 \%$ \\
\hline Large intestine and rectum & 30 & $5.3 \%$ & 23 & $6.0 \%$ & 12 & $7.3 \%$ \\
\hline Gallbladder & 3 & $0.5 \%$ & 1 & $0.3 \%$ & 0 & $0.0 \%$ \\
\hline Appendix & 2 & $0.4 \%$ & 1 & $0.3 \%$ & 0 & $0.0 \%$ \\
\hline Brain (CNS) & 11 & $2.0 \%$ & 6 & $1.6 \%$ & 14 & $8.5 \%$ \\
\hline Meninges & 22 & $3.9 \%$ & 16 & $4.2 \%$ & 0 & $0.0 \%$ \\
\hline Naso- and oropharynx & 13 & $2.3 \%$ & 10 & $2.6 \%$ & 2 & $1.2 \%$ \\
\hline Skin & 14 & $2.5 \%$ & 11 & $2.9 \%$ & 1 & $0.6 \%$ \\
\hline Bone & 6 & $1.1 \%$ & 5 & $1.3 \%$ & 3 & $1.8 \%$ \\
\hline Thyroid gland & 2 & $0.4 \%$ & 1 & $0.3 \%$ & 0 & $0.0 \%$ \\
\hline Thymus & 3 & $0.5 \%$ & 2 & $0.5 \%$ & 0 & $0.0 \%$ \\
\hline Salivary gland & 5 & $0.9 \%$ & 3 & $0.8 \%$ & 0 & $0.0 \%$ \\
\hline Eye & 2 & $0.4 \%$ & 0 & $0.0 \%$ & 2 & $1.2 \%$ \\
\hline Total & 562 & $100.0 \%$ & 382 & $100.0 \%$ & 164 & $100.0 \%$ \\
\hline
\end{tabular}

Abbreviations: TUO Tumor of unknown origin

Table 2 The original diagnoses of the training and validation dataset of TUO

\begin{tabular}{|c|c|c|c|c|c|c|c|c|c|c|c|c|}
\hline \multirow[t]{2}{*}{ Organ } & \multicolumn{4}{|c|}{ Training data } & \multicolumn{4}{|c|}{ Validation data } & \multicolumn{4}{|c|}{ Test data } \\
\hline & No. & $\%$ & Errors & $\%$ & No. & $\%$ & Errors & $\%$ & No. & $\%$ & Errors & $\%$ \\
\hline Primary carcinoma & 232 & $41.3 \%$ & 42 & $18.1 \%$ & 163 & $42.7 \%$ & 25 & $15.3 \%$ & 89 & $54.3 \%$ & 7 & $7.9 \%$ \\
\hline Metastatic carcinoma & 151 & $26.9 \%$ & 39 & $25.8 \%$ & 98 & $25.7 \%$ & 26 & $26.5 \%$ & 19 & $11.6 \%$ & 4 & $21.1 \%$ \\
\hline Benign (normal) lesion & 33 & $5.9 \%$ & 4 & $12.1 \%$ & 22 & $5.8 \%$ & 3 & $13.6 \%$ & 13 & $7.9 \%$ & 1 & $7.7 \%$ \\
\hline Benign mesenchymal tumor & 120 & $21.4 \%$ & 28 & $23.3 \%$ & 80 & $20.9 \%$ & 24 & $30.0 \%$ & 24 & 14.6 & 4 & $16.7 \%$ \\
\hline \multirow[t]{2}{*}{ Malignant mesenchymal tumor } & 26 & $4.6 \%$ & 8 & $30.8 \%$ & 19 & $5.0 \%$ & 6 & $31.6 \%$ & 19 & $11.6 \%$ & 2 & $10.5 \%$ \\
\hline & 562 & $100.0 \%$ & 121 & $21.5 \%$ & 382 & $100.0 \%$ & 84 & $22.0 \%$ & 164 & $100 \%$ & 18 & $11 \%$ \\
\hline
\end{tabular}


tumours (23.3\%), primary carcinoma (18.1\%), and benign (normal) lesion (12.1\%).

\section{Validation data}

The organs and the original diagnoses are shown in Tables 1 and 2. The common organs in the validation dataset were similar to the training dataset, which are lung (19.6\%), liver (11.3\%), kidney (8.1\%), stomach $(5.2 \%)$, and large intestine/rectum (6.0\%) (Table 1). Ascites and peritoneum consist of $5.0 \%$, while pleural fluid and pleura comprised of $4.9 \%$ of the cases (Table 1). Primary carcinoma consists of $42.7 \%$ of the cases, followed by metastatic carcinoma (25.7\%), benign mesenchymal tumour (20.9\%), benign (normal) lesion (5.8\%), and malignant mesenchymal tumour (5.0\%). The hit rate of the presumptive diagnosis of the validation data (top 10) was $78.0 \%$ (Table 3), with the highest error rates at $31.6 \%$ in malignant mesenchymal tumours, followed by benign mesenchymal tumours (30.0\%), metastatic carcinoma (26.5\%), primary carcinoma (15.3\%) and benign (normal) lesion (13.6\%).

\section{Test data}

We exploited 164 patients' cases for the test dataset. The organ and the original diagnoses are shown in Tables 1 and 2. The most common organs were lung (15.9\%), liver (20.1\%), female genital tract including uterus and vulva, vagina $(10.1 \%)$, kidney $(9.1 \%)$, brain $(8.5 \%)$, large intestine and rectum $(7.3 \%)$ and stomach $(5.5 \%)$ (Table 1$)$. Primary carcinoma consists of $54.3 \%$ of the cases, followed by metastatic carcinoma (11.6\%), benign (normal) lesion (7.9\%), benign mesenchymal tumour (14.6\%), and malignant mesenchymal tumour (11.6\%) (Table 2). The hit rate of the presumptive diagnosis of the training data (top 10) was $89 \%$ (Table 3). The error rates being the highest at $21.1 \%$ in metastatic carcinoma, followed by benign mesenchymal tumours (16.7\%), malignant mesenchymal tumours (10.5\%), primary carcinoma (7.9\%), and benign (normal) lesion $(7.7 \%)$.

\section{The precision error rates between training, validation,} and test dataset

The error rates of the precision diagnosis were 21.5 and $22.0 \%$ for training and validation datasets, respectively
(Table 3); which was not significantly different ( $p$-value $=$ $0.866)$. The error rates of the precision diagnosis for test dataset was much less up to $11.0 \%$. The overall hit rate was $79.9 \%$ (Table 3 ).

\section{Example of application}

Let us take an example application of ImmunoGenius in real pathology practice. Recently we experienced a 50year-old woman with a $1.5 \mathrm{~cm}$-sized lung mass in her left upper lobe. She had a history of lumpectomy due to invasive ductal carcinoma 5 years ago. In addition, a 1.5 $\mathrm{cm}$-sized thyroid nodule was found during the assessment. Based on this clinical information, we could hypothesize that this nodule can be primary lung adenocarcinoma, recurrent invasive ductal carcinoma, or metastatic thyroid papillary carcinoma. On $\mathrm{H} \& \mathrm{E}$ staining of needle biopsied sample, the tumor was adenocarcinoma with acinar and papillary pattern and irregular nuclei with frequent indistinctive nucleoli, which can be adenocarcinoma of either primary pulmonary, secondary mammary, and secondary thyroidal origin. In this practical setting, most pathologists would choose to perform IHC for CK7, CK20, TTF-1, GCDFP-15, galectin3, and napsin A for the differential diagnosis. We performed these markers at the first round of IHC and it was positive for CK7, TTF-1, galectin 3 and napsin A, and negative for CK20 and GCDFP-15 (Supplementary Fig. 3) TTF-1 and napsin A are very important markers for the lung cancer diagnosis, GCDFP-15 is important for breast cancer, and CK7, galectin 3, and TTF-1 are important for thyroid cancer diagnosis. However, as we see in the textbook table, napsin A can be also found in 5\% of thyroid cancers as well as galectin 3 can be found up to $50 \%$ of lung adenocarcinoma. Therefore, we can rule out the possibility of breast cancer, but it can either be lung or thyroid carcinoma. So we had to get help from ImmunoGenius application on this case to check the real probabilistic difference calculated by these IHC profiles and the probability of both adenocarcinoma and thyroid carcinoma turned out to be similar as $64 \%$ (Supplementary Fig. 3). For the confirmative diagnosis, we additionally performed the IHC for CK19, thyroglobulin, MOC31, PAX8 and p63. As a result, we could find the most probable diagnosis is lung cancer with $56 \%$ probability and thyroid carcinoma showed $53 \%$ of

Table 3 The comparison of Precision error rates between the training and validation dataset of TUO

\begin{tabular}{|c|c|c|c|c|c|c|c|c|}
\hline \multirow{2}{*}{$\begin{array}{l}\text { Precision } \\
\text { diagnosis }\end{array}$} & \multicolumn{2}{|c|}{ Training data } & \multicolumn{2}{|c|}{ Validation data } & \multicolumn{2}{|c|}{ Test data } & \multicolumn{2}{|l|}{ Total } \\
\hline & No. & $\%$ & No. & $\%$ & No. & $\%$ & No. & $\%$ \\
\hline Accurate results & 441 & $78.5 \%$ & 298 & $78.0 \%$ & 146 & $89 \%$ & 885 & $79.9 \%$ \\
\hline Error results & 121 & $21.5 \%$ & 84 & $22.0 \%$ & 18 & $11 \%$ & 223 & $20.1 \%$ \\
\hline Total & 562 & $100.0 \%$ & 382 & $100.0 \%$ & 164 & $100 \%$ & 1108 & $100.0 \%$ \\
\hline
\end{tabular}

Abbreviations: TUO Tumor of unknown origin 
probability when it is an anaplastic histologic variant (Supplementary Fig. 3). With these results, we could rule out thyroid carcinoma more confidently with presumptive diagnosis prediction by ImmunoGenius.

\section{Discussion}

In the present study, we verified the estimated the diagnostic probability of certain TUOs, using IHC results, by probabilistic decision tree and corresponding mobile application. The precision diagnosis drawn by the probabilistic decision tree algorithm, at the hit rate of $79.9 \%$, can be a convincing assistant in decision making for pathologists. The hit rate rates between training, validation dataset were not statistically significant $(78.5 \%$ vs. $78.0 \%, p$-value $=0.866)$.

The hit rate of the presumptive diagnosis was generally poor compared to the results of our prior validation study using lymphoma cases that showed 95\% precision hit rate [29]. It is mainly due to the magnitude of the disease entities (2009 vs. 104). The common organs in the data used were lung, liver, kidney, ascites and peritoneum, and pleural fluid/pleura where metastatic lesions are often found in clinical practice. In case of the lungs, IHC was commonly used for subtyping between small cell, adeno, and squamous cell carcinoma, as well as determining the origin of the tumour, and whether it is primary or metastatic. In case of the kidneys, IHC was also used for subtyping between clear cell, chromophobe, papillary, etc., as well as determining whether it is primary or metastatic. For ascites and peritoneum, IHC was used for determining whether it is a metastatic carcinoma, or reactive mesothelial cells/macrophages. Moreover, in case of pleural fluid and pleura, IHC was used for determining whether it is metastatic adenocarcinoma (from the lung), mesothelioma, or reactive mesothelial cells/macrophages. Furthermore, in case of stomach, the primary differential diagnosis was between spindle cell neoplasms including gastrointestinal stromal tumours (GIST), schwannoma, and leiomyoma. Finally, in case of colon/rectum, benign spindle cell neoplasms and neuroendocrine cell tumours (carcinoid) were the most common disease.

The primary cause of inaccurate presumptive diagnosis was atypical IHC profiles (compared to that described in the textbook; about two thirds). The major causes of inaccurate presumptive diagnosis included overlapping IHC profiles between adenocarcinomas of the gastrointestinal tract, the origin of squamous cell carcinoma (no sitespecific marker for squamous cell carcinoma), mesenchymal neoplasia that express both epithelial and mesenchymal markers, tumours with mixed or combined entities (e.g. squamous transformation of adenocarcinoma of the lung after chemotherapy, combined germ cell tumour, etc.), and tumours with no disease-specific markers. The cases with typical IHC markers tended to show accurate presumptive diagnosis. In other words, the precise differential diagnosis cannot be made only using the IHC profile in about $22 \%$ of the cases, and clinicopathologic findings along with the patient history should be considered. Thus, this algorithm should be used and interpreted with contextual information in a comprehensive and integrated manner. This study clearly showed the feasibility and clinical utility of making a diagnosis using the probabilistic decision tree algorithm and iOS and Android mobile application in the differential diagnosis of the tumours using IHC profiles.

\section{Conclusions}

The overall hit rate of this machine-learning algorithm was $79.9 \%$, and the hit rate rates were not significantly different between training and validation data, and it was much lower in test data, thus showing a relatively robust generalization. Disease-specific markers, overlapping IHC profiles between diseases, a lack of site-specific markers, mixed/combined tumours, and atypical IHC profile are the leading causes of error in this system. However, this system will be useful to assist the pathologists in making precise decisions during the disease diagnosis Integrated interpretation with contextual information such as clinical and pathological findings should be considered, along with the use of this application, before making a final decision. Further studies for recommending IHC panels for particularly complex problems regarding differential diagnosis and application of artificial neural network algorithms to optimize the disease diagnosis [30, 31], organ incidence, and antibody weight are needed in the future.

\section{Abbreviations}

IHC: Immunohistochemistry; WHO: World Health Organization; TUOs: Tumours of unknown origin; GIST: Gastrointestinal stromal tumours

\section{Supplementary Information}

The online version contains supplementary material available at https://doi. org/10.1186/s13000-021-01081-8.

Additional file 1: Figure S1. The example of the $\mathrm{IHC}$ database.

Additional file 2: Figure S2. The example of the patients $\mathrm{HC}$ profile dataset for training and validation of the diagnosis presumption algorithm.

Additional file 3: Figure S3. The example of application using a case of tumor of unknown origin.

Additional file 4: Table S1. The reference books used for $\mathrm{HC}$ database build. Table S2. Online references used for IHC antibody name documentation.

Acknowledgments

We appreciate Mr. Young Dong Seo for reviewing the manuscript style.

Patents

Copyright of ImmunoGenius, the mobile application developed during this project, is owned by The Catholic University of Korea, Indurstry-Academic Cooperation Foundation. The content of the software including the idea, database, user interface, and the source code is protected. 


\section{Authors' contributions}

Conceptualization, YC, HY; methodology, YC, MYC, and HY; software, YC.; validation, YC., and N.T.; formal analysis, YC.; investigation, YC, JC, YK; resources, YC, data curation, YC, writing —original draft preparation, $Y C$.; writing - review and editing, YC, MYC and NT.; visualization, YC, NT. supervision, MYC, HY... funding acquisition, YC, JC, YK. The authors read and approved the final manuscript

\section{Funding}

This research was supported by Basic Science Research Program through the National Research Foundation of Korea (NRF) funded by the Ministry of Education (2016R1D1A1A02937427), partly funded by a research grant from the institute of Clinical Medicine Research, Catholic University of Korea, Yeouido St. Mary's Hospital, supported by the Po-Ca Networking Groups funded by the Postech-Catholic Biomedical Engineering Institute (PCBMI) (No 5-2016-B0001-00149).

\section{Availability of data and materials}

The datasets used and/or analyzed during the current study are available from the corresponding author on reasonable request.

\section{Declarations}

\section{Ethics approval and consent to participate}

The present study was approved by the ethical review committee of Institutional Review Board of the Catholic University of Korea, College of Medicine. Written informed consent was obtained from all enrolled patients.

\section{Consent for publication}

Not applicable.

\section{Competing interests}

The authors declare that they have no competing interests.

\section{Author details}

'Department of Hospital Pathology, College of Medicine, The Catholic University of Korea, 271, Cheonbo-ro, Uijeongbu 11765, Gyeonggi-do, Republic of Korea. ${ }^{2}$ Postech-Catholic Biomedical Engineering institute, College of Medicine, The Catholic University of Korea, Seoul, Republic of Korea. ${ }^{3}$ Dasom X, Inc., Seoul, Republic of Korea. ${ }^{4}$ Department of Creative Information Technology, POSTECH, Pohang, Republic of Korea. ${ }^{5}$ University of Texas Health Science Center, Houston, TX, USA. ${ }^{6}$ Computer Science and Engineering, POSTECH, Pohang, Republic of Korea. ${ }^{7}$ Department of Pathology, Yonsei University, Wonju College of Medicine, Wonju, Republic of Korea.

Received: 11 August 2020 Accepted: 1 March 2021

Published online: 11 March 2021

\section{References}

1. Elias JM. Immunohistochemistry : a brief historical perspective : commentary. Natick: Eaton Pub.; 2000

2. Buchwalow IB, Böcker W. Immunohistochemistry : basics and methods. Heidelberg: Springer; 2010

3. Matos LL, Trufelli DC, de Matos MG, da Silva Pinhal MA Immunohistochemistry as an important tool in biomarkers detection and clinical practice. Biomark Insights. 2010;5:9-20.

4. Chu PG, Weiss LM. In: 2nd edn, editor. Modern immunohistochemistry. Cambridge: Cambridge University Press; 2014.

5. Dabbs DJ. Diagnostic immunohistochemistry : theranostic and genomic applications. 4th ed. Philadelphia: Elsevier/Saunders; 2014.

6. Kalyuzhny AE. Immunohistochemistry : essential elements and beyond. Cham: Springer; 2016.

7. Coons AH. Labeling techniques in the diagnosis of viral diseases. Bacteriol Rev. 1964;28:397-9.

8. Werner B, Campos AC, Nadji M, Torres LFB. Practical use of immunohistochemistry in surgical pathology. J Bras Patol Med Lab. 2005; 41(5):353-64.

9. DeYoung BR, Wick MR. Immunohistologic evaluation of metastatic carcinomas of unknown origin: an algorithmic approach. Semin Diagn Pathol. 2000;17(3):184-93.
10. Bishop JA, Sharma R, Illei PB. Napsin a and thyroid transcription factor-1 expression in carcinomas of the lung, breast, pancreas, colon, kidney, thyroid, and malignant mesothelioma. Hum Pathol. 2010;41(1):20-5.

11. Yu H, Li L, Liu D, Li WM. Expression of TTF-1, NapsinA, P63, CK5/6 in lung Cancer and its diagnostic values for histological classification. Sichuan Da Xue Xue Bao Yi Xue Ban. 2017;48(3):336-41.

12. El-Maqsoud NM, Tawfiek ER, Abdelmeged A, Rahman MF, Moustafa AA. The diagnostic utility of the triple markers Napsin a, TTF-1, and PAX8 in differentiating between primary and metastatic lung carcinomas. Tumour Biol. 2016;37(3):3123-34.

13. Gweon HM, Kim JA, Youk JH, Hong SW, Lim BJ, Yoon SO, Park YM, Son EJ. Can galectin-3 be a useful marker for conventional papillary thyroid microcarcinoma? Diagn Cytopathol. 2016;44(2):103-7.

14. Kandalaft PL, Gown AM. Practical applications in immunohistochemistry: carcinomas of unknown primary site. Arch Pathol Lab Med. 2016;140(6):508-23.

15. Lin F, Prichard J. Handbook of practical immunohistochemistry : frequently asked questions. 2nd ed. New York: Springer; 2015.

16. Lesaffre E, Lawson A. Bayesian biostatistics. Chichester: Wiley; 2012.

17. Aaltonen LA, Hamilton SR, World Health Organization., International Agency for Research on Cancer. Pathology and genetics of tumours of the digestive system. Lyon: IARC Press; 2000.

18. DeLellis RA, International Agency for Research on Cancer., World Health Organization., international academy of pathology., International Association for the Study of Lung Cancer. Pathology and genetics of tumours of endocrine organs. Lyon: IARC Press; 2004.

19. Travis WD, World Health Organization., International Agency for Research on Cancer., International Association for the Study of Lung Cancer., International Academy of Pathology. Pathology and genetics of tumours of the lung, pleura, thymus, and heart. Lyon: IARC Press; 2004

20. LeBoit PE, International Agency for Research on Cancer., World Health Organization., International Academy of Pathology., European Organization for Research on treatment of Cancer., UniversitätsSpital Zürich. Departement Pathologie. Pathology and genetics of skin tumours. Lyon: IARC Press; 2006.

21. Louis DN, International Agency for Research on Cancer., World Health Organization. WHO classification of tumours of the central nervous system. Lyon: International Agency for Research on Cancer; 2007.

22. Swerdlow SH, International Agency for Research on Cancer., World Health Organization. WHO classification of tumours of haematopoietic and lymphoid tissues. 4th ed. Lyon: International Agency for Research on Cancer; 2008.

23. Rekhtman N, Bishop JA. Quick reference handbook for surgical pathologists. Heidelberg: Springer; 2011

24. Lakhani SR, International Agency for Research on Cancer., World Health Organization. WHO classification of tumours of the breast. Lyon: International Agency for Research on Cancer; 2012

25. Fletcher CDM, World Health Organization., International Agency for Research on Cancer. WHO classification of tumours of soft tissue and bone, 4th edn. Lyon: IARC Press; 2013.

26. International Agency for Research on Cancer (IARC), Moch H. WHO classification of tumours of the urinary system and male genital organs. 4th ed. Lyon: International Agency for Research on Cancer; 2016.

27. Louis DN, International Agency for Research on Cancer. WHO classification of tumours of the central nervous system, revised 4th edn. Lyon: International Agency For Research On Cancer; 2016.

28. ImmunoQuery [Internet] Philadelphia: Elsevier; 2020. [cited 2020 Mar 1]. Available from: [http://www.immunoquery.com].

29. Chong Y, Lee JY, Kim Y, Choi J, Yu H, Park G, Cho MY, Thakur N. A machinelearning expert-supporting system for diagnosis prediction of lymphoid neoplasms using a probabilistic decision-tree algorithm and immunohistochemistry profile database. J Pathol Transl Med. 2020;54(6): 462-70.

30. Thakur N, Yoon H, Chong Y. Current trends of artificial intelligence for colorectal Cancer pathology image analysis: a systematic review. Cancers. 2020;12(7):1884

31. Nam S, Chong Y, Jung CK, Kwak TY, Lee JY, Park J, Rho MJ, Go H. Introduction to digital pathology and computer-aided pathology. J Pathol Transl Med. 2020;54(2):125-34.

\section{Publisher's Note}

Springer Nature remains neutral with regard to jurisdictional claims in published maps and institutional affiliations. 